\title{
Experimental evidence of ultrasonic stimulation of brine-oil flow through a porous rock in laboratory conditions
}

\author{
Xiaoyun Tu • Gijsbert Ooms • Fred van der Bas
}

Received: 2 November 2005 / Accepted: 12 January 2007 / Published online: 22 February 2007

(C) Springer Science+Business Media B.V. 2007

\begin{abstract}
Laboratory experiments have been carried out to study the influence of high-frequency $(20 \mathrm{kHz})$ acoustic waves on the flow of brine through a core sample of reservoir rock containing residual oil. The experiments were done at reservoir conditions with respect to temperature and pressure. Below a certain critical level of the acoustic intensity the relative permeability for the flow of brine is independent of the acoustic intensity and there is no flow of oil out of the core. However, above the critical level the relative permeability for the brine flow jumps to a significantly higher value and oil starts to flow from the core. These experimental results are in agreement with a theoretical model proposed by Graham and Higdon [Oscillatory flow of droplets in capillary tubes Part 2 Constricted tubes. J. Fluid Mech. 425, 55-77 (2000)]. The model is based on a microscopic picture of oil drops (surrounded by flowing brine) being trapped inside the pores of the reservoir rock. Under the influence of ultrasonic waves the oil drops oscillate and can escape from their pores above a critical level of the acoustic intensity.
\end{abstract}

\section{Introduction}

In order to increase both the production rate and the total amount of oil that can be withdrawn from an oil reservoir a number of techniques can be applied. The ensemble of these techniques is called stimulation. Classical stimulation techniques are

X. Tu · G. Ooms $(\bowtie)$

J. M. Burgers Centre, Delft University of Technology, Laboratory for Aero- and Hydrodynamics, Leeghwaterstraat 21, 2628 CA Delft, The Netherlands

e-mail: g.ooms@tudelft.nl

$\mathrm{X} . \mathrm{Tu}$

e-mail: xiaoyun.tu@tno.nl

F. van der Bas

Shell International Exploration and Production, Technology Application and Research, P.O. Box 60, 2280 AB Rijswijk, The Netherlands 
injection of acid or solvent, injection of steam and hydraulic fracturing. New stimulation techniques have been proposed and investigated. Tambini reviews these techniques and he mentions, among others, acoustic stimulation. In this publication we concentrate on the influence of high-frequency acoustic waves on the flow of brine through a porous rock containing residual oil.

The first laboratory study regarding the influence of ultrasonic waves on the flow of brine and oil through a porous material was carried out by Duhon (1964). He studied the characteristics of oil displacement by brine in the presence of ultrasound and observed an increase in oil recovery from sandstone as a result of the ultrasonic excitation. Gadiev (1977) conducted laboratory experiments in saturated, unconsolidated sand that was exposed to vibration with frequencies between $40 \mathrm{~Hz}$ and $15 \mathrm{kHz}$. As result of the excitation an increase of $10-15 \%$ of oil displacement by brine was observed. The effect of ultrasound on the displacement of oil and brine in a porous material was also studied by Neretin and Yudin (1981). The oil yield increased by a factor ranging from $65 \%$ to $85 \%$.

Beresnev and Johnson (1994) concluded after a careful review of the literature, including also publications in Russian not easily accessible, that no clear evidence was available about the mechanisms responsible for the flow stimulation by acoustic irradiation. They suggested that the following mechanisms might be involved: (1) the acoustic wave field may considerably reduce the influence of capillary forces on the oil percolation, resulting in an increased rate of migration through the porous material, (2) non-linear effects such as acoustic streaming, may give the liquids extra momentum, (3) reduction of the surface tension and the viscosity of the liquids due to a temperature increase caused by energy dissipation may result in an apparent change in permeability, and (4) mechanical abrasion effect on pores may be a consequence of high frequency and high intensity acoustic fields.

Graham and Higdon (2000) investigated the motion of a fluid drop in a constricted capillary tube filled with a different fluid for flows subject to the combined action of a mean pressure gradient and an oscillatory body force. They employed numerical calculations to determine the effect of the oscillatory forcing on the flow rate of the continuous fluid and the movement of the drop. It is the purpose of this publication to determine the validity of Graham and Higdon's model for the understanding of the influence of high-frequency $(20 \mathrm{kHz})$ acoustic waves on the flow of brine through the pores of a core sample of reservoir rock containing residual oil drops. Results will be shown of acoustic-stimulation experiments performed at reservoir conditions with a constant flow rate of brine and at increasing intensity of high-frequency acoustic waves applied to the core. Special attention will be paid to the relative permeability of the brine flow as a function of the acoustic intensity.

\section{Oscillatory movement of an oil drop in a pore under the influence of an acoustic wave}

After the conventional water flood processes the residual oil in the near wellbore region of a reservoir normally remains as a discontinuous phase in the form of oil drops in the continuous brine phase. These oil drops are trapped in the pores of the reservoir rock by capillary forces. This residual oil can be of the order of $70 \%$ of the original oil in place. In Fig. 1 a possible microscopic picture is sketched for a single oil drop trapped inside a pore of the porous rock (assumed to be water wettable). 


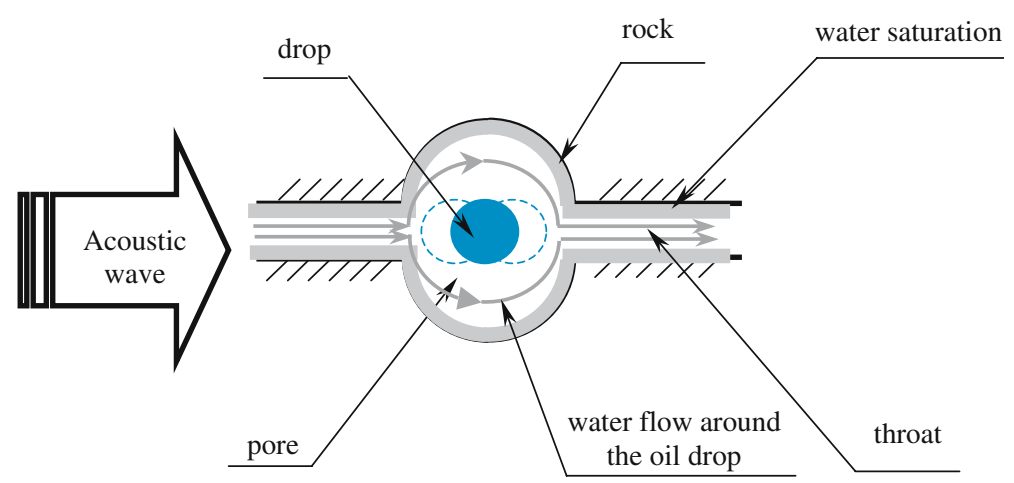

Fig. 1 Oil drop trapped inside a pore for the case that both the mean pressure gradient in the brine and the acoustic intensity are too small to drive the oil drop through the pore throat. The brine flows from left to right through the gap between the surface of the oil drop and the pore walls into the pore throat

The oil drop is surrounded by brine flowing through the gap between the surface of the oil drop and the pore walls. Without acoustic stimulation it is assumed that the brine flow is not strong enough to push the drop through the pore throat. When an acoustic wave is applied the oil drop starts to oscillate. At low acoustic intensity the oscillation will still not be strong enough to push the drop through the throat of the pore. However it is assumed that with increasing acoustic intensity the oscillation can become so strong, that the drop penetrates into the pore throat and passes it. So at a certain critical intensity there is a sudden change in the flow, also at the macroscopic level. Oil will start to flow out of the porous rock and at a constant brine flow rate the pressure drop will decrease, as the obstructing oil drops are removed.

As mentioned quantitative calculations concerning the motion of an oil drop in a constricted pore filled with brine for flows subject to the combined action of a mean pressure gradient and an acoustic body force were given in a publication by Graham and Higdon (2000). They employed numerical calculations to determine the effect of an oscillatory forcing on the flow rate of brine and the movement of the oil drop. The following two paragraphs are taken (with some small adaptations) from their publication.

In the absence of oscillatory forcing a critical pressure gradient for drop propagation exists, below which the drop becomes plugged in the narrow constriction of the pore. In Fig. 2 (Figure 15 in the publication of Graham and Higdon) the dimensionless mean flow rate of brine $\bar{U} / \bar{U}_{1}$ is shown as a function of the dimensionless oscillatory forcing level $F_{\text {rms }}$ due to the acoustic wave. For this plot the mean pressure gradient is below the threshold required to push the drop through the constriction and the dimensionless acoustic frequency is low $\left(L / \bar{U}_{1} \tau \rightarrow 0\right)$. $\bar{U}$ represents the mean flow rate of brine through the pore and $\bar{U}_{1}$ the mean flow rate for the case of a brine flow without an oil drop in the pore (all other conditions being the same). $L$ represents the length of the pore and $1 / \tau$ the frequency of the acoustic wave. $F_{\text {rms }}$ is defined as

$$
F_{\mathrm{rms}}=F_{\omega} / \sqrt{2}=G_{\omega} h^{2} /(\gamma \sqrt{2}),
$$

in which $G_{\omega}$ represents the oscillatory body force, $h$ the radius of the pore neck and $\gamma$ the surface tension. For low levels of oscillating forcing, the flow rate is very small due to pore plugging by the oil drop. As the oscillatory forcing level increases, a point is 
Fig. 2 Dimensionless mean flow rate of brine as a function of the oscillatory forcing level due to an acoustic wave for the case that the mean pressure gradient is below the threshold required to push the oil drop through the constriction and assuming that the acoustic frequency is low

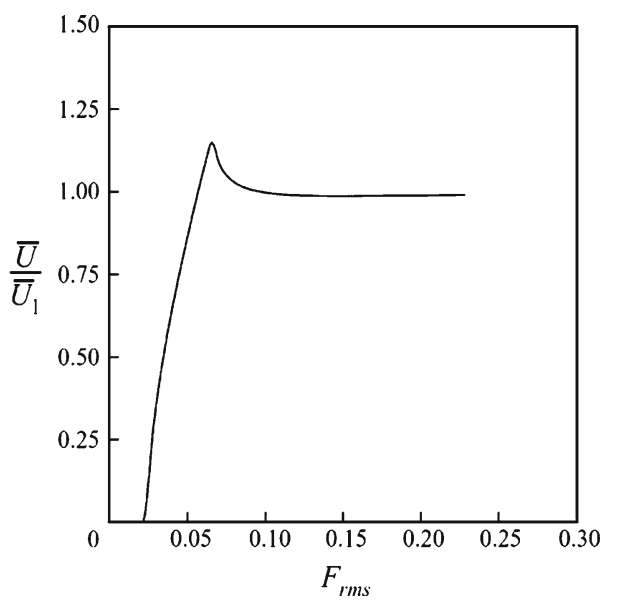

reached at which the instantaneous force is strong enough to drive the drop through the constriction. Once this threshold is reached, the flow rate of brine increases dramatically. Further increases in the forcing level cause the mean flow rate to decrease slightly before levelling off. The shape of this plot can be understood in the context of Fig. 3 (Figure 16 in the publication of Graham and Higdon), which shows an idealized version of the mean velocity of brine as a function of a constant forcing level. In regions 1 and 4 the permeability is constant (velocity vs. force is linear), and in regions 2 and 3 plugging occurs. For the quasi-steady $\left(L / \bar{U}_{1} \tau \rightarrow 0\right)$ results shown in Fig. 2 , the steady component of the force (the mean pressure gradient) is in region 2 (plugging), and the sharp increase in mean flow rate occurs when the positive portion of the oscillatory forcing cycle (the acoustic wave) samples region 1 (linear) and the negative portion samples region 3 (plugging). As the oscillatory forcing becomes stronger, the negative portion of the forcing samples region 4 (linear) and the mean flow rate decreases. For strong oscillatory forcing, the mean bulk velocity is comparable to the flow rate for a single-phase liquid at the same constant forcing level of the mean pressure gradient and no oscillatory forcing. This indicates that the addition of the oscillatory forcing to a plugging capillary flow not only mobilizes the drop but also reduces the drop resistance enough to generate a significant mean flow rate.

Having shown that significant positive flow enhancement occurs for oscillatory forcing levels above a certain critical value of the acoustic intensity, Graham and Higdon then focus on how this critical value depends on the acoustic frequency. Figure 4 (Figure 22 in the publication of Graham and Higdon) shows their computed values of the dimensionless critical frequency as a function of the oscillatory forcing level. At a certain forcing level the drop moves freely through the constriction for frequencies below a critical value of the frequency, but is immobilized for frequencies above the critical value. To understand the behavior seen in Fig. 4, the mechanism by which the oscillatory forcing remobilizes the drop must be examined. A drop which is trapped on the upstream side of a constriction must be displaced from its original position to a point just past the midpoint of the constriction. This distance is a function of the surface tension and the capillary geometry but scales linearly with the length scale $L$. For oscillatory forcing levels significantly above plugging, the instantaneous drop velocity is nearly proportional to $F_{\omega}$ even in the narrow constriction. The amplitude of 
Fig. 3 Idealized version of the mean velocity of brine as a function of a constant forcing level

Fig. 4 Computed values of the dimensionless critical frequency as a function of the oscillatory forcing level. An oil drop moves freely through the constriction for frequencies below the critical value of the frequency, but is immobilized for frequencies above it
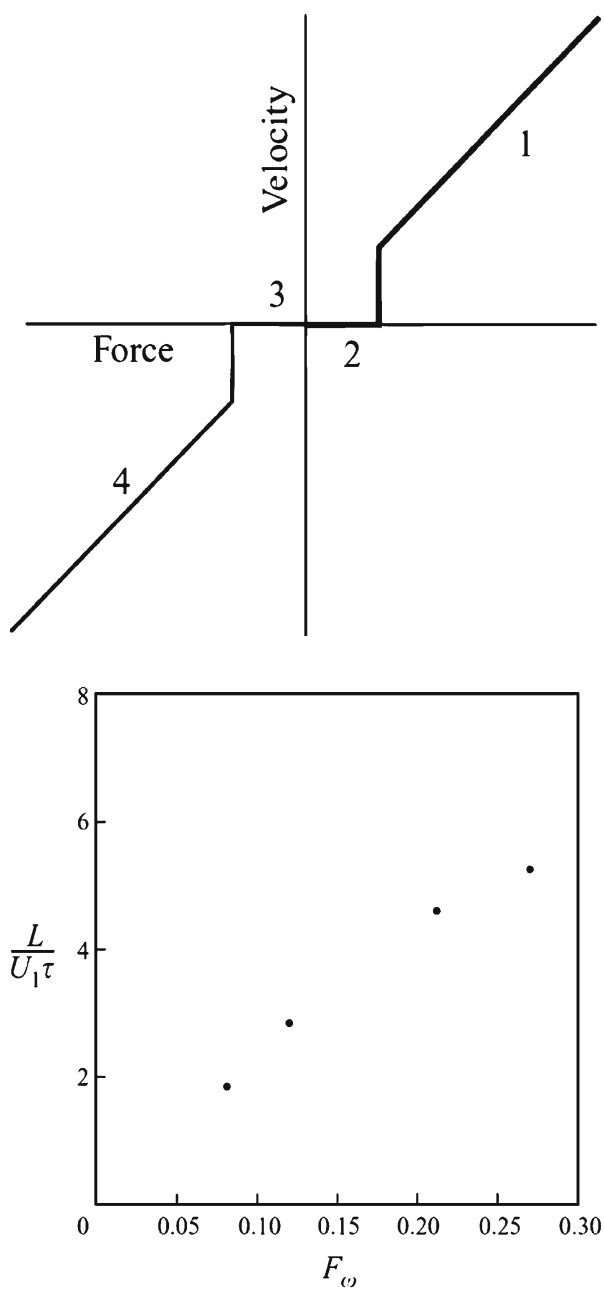

the oscillatory displacement thus scales as $F_{\omega} \tau$. Equating this displacement with the distance required for remobilization yields a linear scaling for the critical frequency $L / \bar{U}_{1} \tau$, which is consistent with the results shown in Fig. 4. In our work we are in particular interested in the influence of high-frequency waves $(20 \mathrm{kHz})$ on the flow of brine and oil through a sample of reservoir rock. Based on the results given in Fig. 4 we may expect significantly higher values of the acoustic forcing $F_{\omega}$ necessary for flow enhancement than the values shown in the figure. The results of Graham and Higdon presented so far are based on their quasi-steady analysis $\left(L / \bar{U}_{1} \tau \rightarrow 0\right)$. However in their publication they also give the results of full unsteady flow simulations. There is good agreement between the quasi-steady predictions and the unsteady simulations.

It is pointed out, that Graham and Higdon do not consider damping of the acoustic wave. As mentioned, in our work we are in particular interested in the influence of high-frequency waves $(20 \mathrm{kHz})$ on the flow of brine and oil through a sample of reservoir rock and damping can become important already at relatively short distances (say $0.2 \mathrm{~m}$ ). 


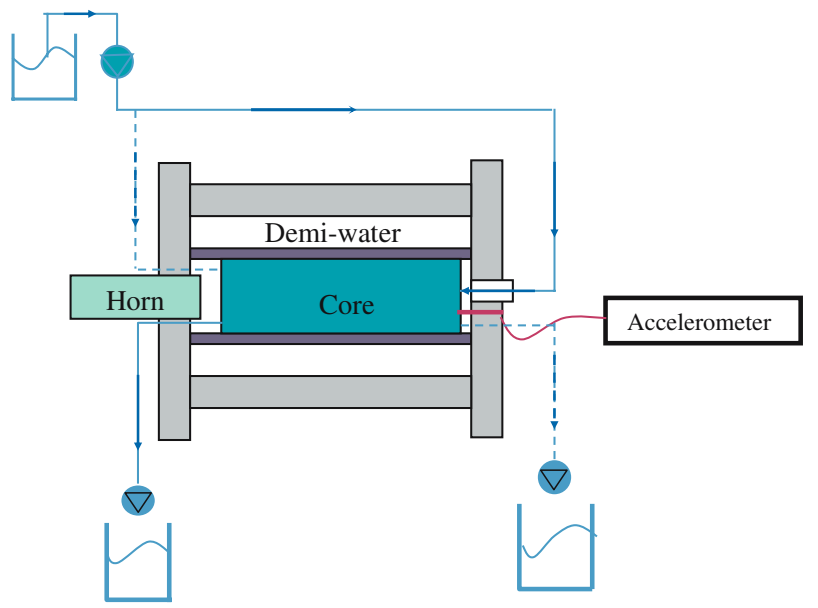

Fig. 5 Experimental set-up. The core is placed in a rubber sleeve inside the steel vessel. The space between the vessel and sleeve, which is filled with demineralized water, is pressurized to seal the sample. On the left is the acoustic horn that generates the high-frequency acoustic wave. On the right is the accelerometer which can be used to measure the amplitude and hence the damping of the acoustic signal after passage through the core

\section{Experimental set-up and measurements}

The experiments were carried out in the set-up shown in Fig. 5. It is the same as used by Poesio et al. (2002). The core that was used for the experiments was a cylindrically shaped Berea sandstone sample. The length of the core was $20 \mathrm{~cm}$ and the diameter $7.62 \mathrm{~cm}$. The porosity was about 0.25 . During the experiment the core was placed in a rubber sleeve to keep it fixed. It was then placed in a steel vessel in which down-hole reservoir conditions were simulated (up to 150 bar and $100^{\circ} \mathrm{C}$ ). An acoustic horn was placed at one end of the core. The high pressure in the vessel made it possible to avoid cavitation (for pressures lower than 100 bar the influence of cavitation becomes noticeable). The space between the vessel and sleeve, which was filled with water, was pressurized to 180 bar to make the rubber sleeve completely sealed off.

There were four pressure sensors, two along the core (at $2.54 \mathrm{~cm}$ and $10.70 \mathrm{~cm}$ ) and two at both ends of the core, dividing the core into three sections. The pressure drop across each section was monitored and recorded. To measure the temperature at two locations in the core two thermocouples were installed at the sidewall of the porous medium, through the rubber sleeve. Also the temperature at the front and at the back of the core could be measured. The data were sent to a digital data recorder and processed on a computer.

The ultrasonic equipment consisted of: (1) a converter which converted electricity into mechanical vibrations of a piezoelectric element, (2) an amplifier which was used to set the amplitude of the vibrations, and (3) an ultrasonic horn (a Branson Module PGA $22020 \mathrm{kHz}$ horn with maximum power output of $2 \mathrm{~kW}$ ) which concentrated the mechanical vibrations onto the front side of the core sample. An accelerometer was placed at the end of the core opposite to the ultrasonic horn (see Fig. 5). It was used to measure the amplitude of the acoustic signal after passage through the core. In this way the damping of the signal was determined during the experiments. With the 
aid of a system of valves the flow could be sent either in the same or in the opposite direction of the traveling acoustic waves. During all the experiments reported here the flow was kept in the opposite direction of the acoustic waves. From the explanation of acoustic stimulation based on Fig. 3 it is clear, however, that the results would be the same if the fluid flow and acoustic wave propagation were in the same direction.

Before performing the acoustic-stimulation experiments the following steps were taken: (1) the core was flushed with $\mathrm{CO}_{2}$ to expel all air in the porous material, (2) after the gas flow was stopped a constant flow rate of the brine was started, and (3) the initial permeability of the core was measured. The salt $(\mathrm{KCl})$ concentration in the brine was $2 \%$. The permeabilities in the three sections of the core (defined above) after brine saturation were equal to $230 \mathrm{mD}, 153 \mathrm{mD}$, and $131 \mathrm{mD}$, respectively. Thereafter oil was injected, which caused a large volume concentration of oil in the core. Finally only brine was injected and a part of the oil was pushed out of the core again. The remaining part of the oil caused a two-phase brine-oil saturation in the core. The final permeabilities were considerably lower than the initial ones, namely $48 \mathrm{mD}, 26 \mathrm{mD}$, and $23 \mathrm{mD}$, respectively. The residual oil saturation after the secondary brine injection was 0.25 . This was determined by means of resistivity measurements.

Thereafter the acoustic-stimulation experiment was carried out. The brine flow rate was fixed at a value of $75 \mathrm{ml} / \mathrm{min}$. Without acoustic stimulation there was no net flow of oil out of the core. Then the acoustic horn was switched on at a low intensity $\left(F_{\omega}=1.38\right.$; an intensity considerably lower than the one at which oil starts to flow out of the core) and the pressure drop over the middle part of the core and temperature inside the core were monitored. We selected the middle part of the core in order to avoid the influence of inlet- or outlet-conditions as much as possible. The temperature increased with time due to the acoustic energy dissipation in the core. As the viscosities of brine and oil (and their surface tension) are dependent on the temperature we will present the results for the pressure drop over the core as function of temperature, not as function of time, in the next section. In this manner it is possible to compare the results of different experiments at the same temperature. (It is important, however, to realize that for an experiment with a constant acoustic power input, increasing temperature means increasing time. Poesio et al. (2002) developed a detailed theoretical model for the calculation of the temperature distribution inside the core as function of time during acoustic irradiation of the core. For more information and details we refer to this publication. The room temperature was constant.) During the experiment it was also monitored, whether oil was flowing out of the core but the flow rate was not measured. After the first stimulation experiment at low acoustic intensity we waited until the core had cooled down to ambient temperature. Thereafter a new stimulation experiment was performed at a higher acoustic intensity. In total, five intensities with increasing values were applied.

\section{Comparison between theoretical model and experiments}

In Fig. 6 the (dimensionless) pressure drop $F_{0}$ over the middle section of the core is shown as a function of temperature and for the five values of the (dimensionless) acoustic intensity $F_{\omega}$. The dimensionless pressure drop is defined by $F_{0}=\Delta P_{0} h^{2} / \gamma$. $\Delta P_{0}$ is the pressure gradient over the middle section of the core; all other quantities 


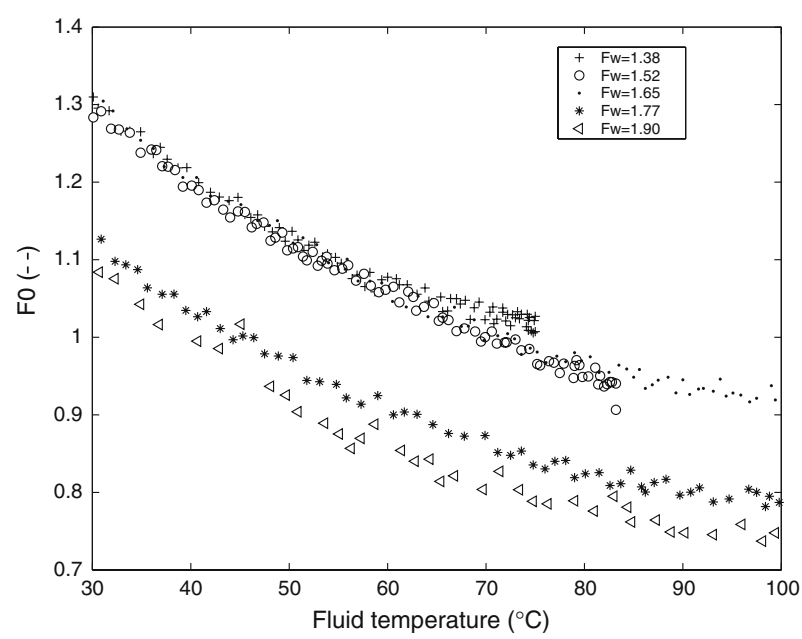

Fig. 6 Dimensionless pressure drop over the middle section of the core as a function of temperature for five values of the dimensionless acoustic intensity. The pressure drop is independent of the acoustic intensity for the three lowest values of this intensity. However, at a critical value of the acoustic intensity the pressure drop is suddenly considerably lower than its value for the first three experiments at the same temperature (over the complete range of temperature values). At this critical value also oil starts to flow out of the core

have been defined earlier. As can be seen from the figure for each particular experiment the pressure drop decreases with increasing temperature, as expected because of the decrease in viscosity of the liquids with increasing temperature (See also Poesio et al. 2002). At the same temperature the pressure drop is independent of the acoustic intensity for the three lowest values of this intensity. However, at a critical value of the acoustic intensity the pressure drop is suddenly considerably lower than its value for the first three experiments at the same temperature (over the complete range of temperature values). At this critical value also oil starts to flow out of the core. With a further increase in acoustic intensity there is a further more gradual decrease of the pressure drop at the same temperature.

This result is in agreement with our expectations based on the arguments given in the preceding section. At low acoustic intensity the oscillation of the oil drops (being trapped inside pores) is not strong enough to enable the drops to penetrate into the pore throats and pass them. At constant brine flow the pressure drop over the core is then relatively insensitive to the strength of the acoustic stimulation. However with increasing acoustic intensity the oscillation of the drops becomes so strong, that at a certain critical intensity they can pass the largest pore throats. Oil will then suddenly start to flow out of the rock and at a constant brine flow rate the pressure drop over the core will decrease suddenly and significantly. As expected the acoustic forcing $F_{\omega}$ necessary for the jump in permeability is for our experiments significantly larger $\left(F_{\omega} \sim 1.7\right)$ than the values shown in Fig. 4, because of the high-frequency acoustic waves that we applied. With further increase in acoustic intensity the oil drops start to pass also through the smaller pore throats, leading to a further decrease in pressure drop (at the same temperature).

Using the measured results for the pressure drop and the temperature we have also calculated the relative permeability of the core for the brine flow during the 


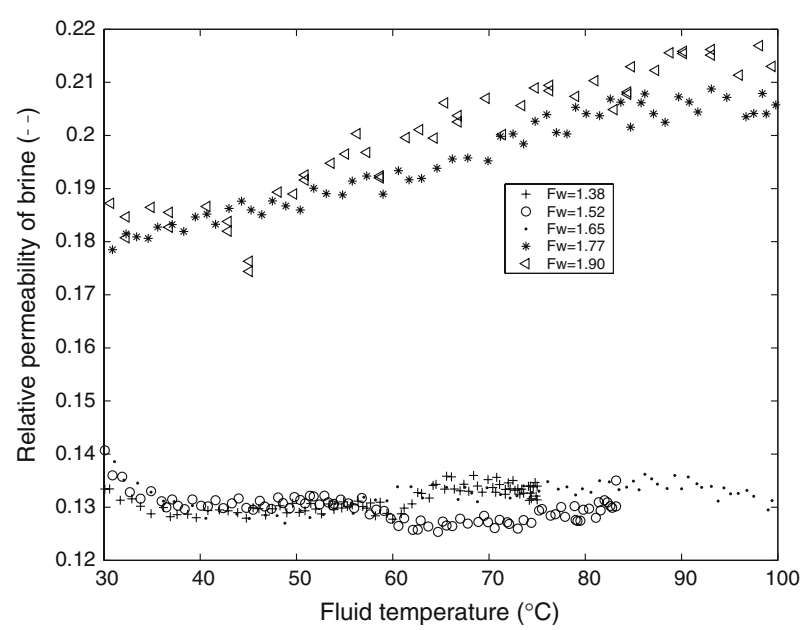

Fig. 7 Relative permeability of the core for the brine flow during the brine-oil flow experiments as function of temperature for five values of the dimensionless acoustic intensity

experiments for each acoustic power input to see how it changes as a consequence of the stimulation. The influence of the temperature on the viscosity was incorporated in the calculation. The results are given in Fig. 7, which shows the relative permeability (for each of the five values of the dimensionless applied acoustic intensity) as a function of the temperature. Below the critical acoustic intensity the relative permeability remains nearly constant as a function of the acoustic intensity and as a function of the temperature. However, when the acoustic intensity becomes larger than its critical value the relative permeability jumps to a higher value and thereafter continues to grow with increasing acoustic intensity (at the same temperature). It is important to point out that although below the critical acoustic intensity the temperature of the liquids increases due to energy dissipation, no increase in relative permeability is observed. So the increase in temperature is not responsible for the jump in relative permeability at the critical acoustic intensity. It is the acoustic stimulation itself, that causes it.

As a reference we carried out similar experiments for the one-phase flow of brine only through the core. The results are given in Fig. 8. As expected the pressure drop for the brine flow experiments is much lower compared to the brine-oil flow experiments (see Fig. 6), as oil drops impeding the brine flow in the pores are not present. Also a critical value for the acoustic intensity above which the pressure drop suddenly decreases, does not occur. As mentioned before Poesio et al. (2002) proved, that the decrease in pressure drop with increasing temperature is due to the decrease in brine viscosity with increasing temperature.

\section{Discussion}

An important simplification in the model of Graham and Higdon is, that they assume the pores to be almost completely blocked by oil drops below the critical value of the acoustic intensity. So in their simulations almost no flow of brine is possible below 


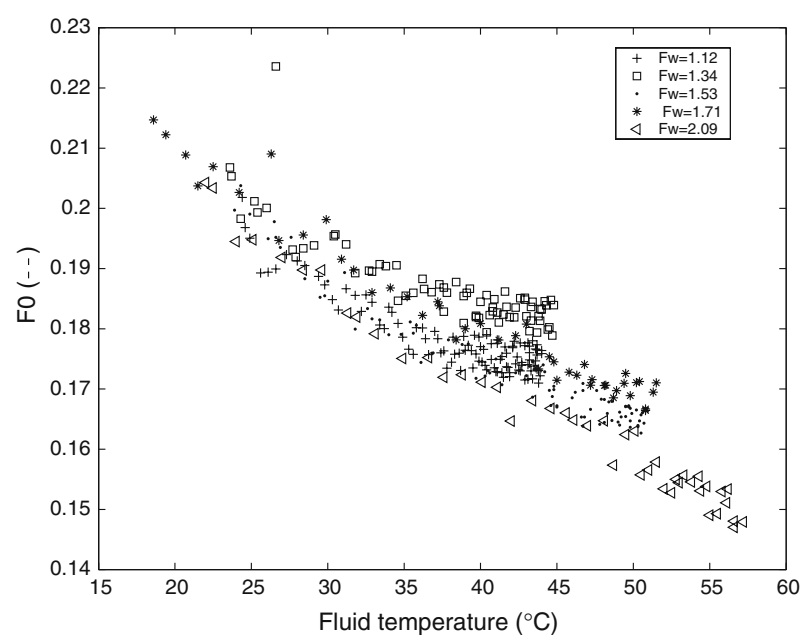

Fig. 8 Dimensionless pressure drop over the core for the one-phase flow of brine only as function of temperature for five values of the dimensionless acoustic intensity

the critical intensity. This is obviously not correct, as we may conclude from our experiments. The reason is likely, that in a porous rock pores of different size are present. Some of them may be blocked by oil drops, whereas larger ones may still be open. Moreover Graham and Higdon do not include damping of the acoustic waves in their model and it is known that wave damping becomes strong at higher frequencies. Nevertheless, our experiments indicate that the model of Graham and Higdon gives a rather realistic qualitative description of the influence of high-frequency acoustic waves on the flow of brine with oil drops through a reservoir rock at underground pressures and temperatures. It is important to point out again that although below the critical acoustic intensity the temperature of the liquids increases due to energy dissipation, no increase in relative permeability is observed. So the increase in temperature is not responsible for the jump in relative permeability at the critical acoustic intensity. It is the acoustic stimulation itself, that causes it. For a more detailed confirmation of the model of Graham and Higdon a visualization of the effect of acoustic stimulation would be very helpful. With our experimental set-up designed for measurements at reservoir pressures and temperatures this is not possible. However, a transparent set-up at ambient pressure and temperature could be used for this purpose.

\section{References}

Graham, D.R., Higdon, J.J.L.: Oscillatory flow of droplets in capillary tubes. Part 2. Constricted tubes. J. Fluid Mech. 425, 55-77 (2000)

Tambini, M.: Beyond acidizing and fracturing, SPE European Formation Damage Conference, SPE 82573

Duhon, R.: An investigation of the effect of ultrasonic energy on the flow of fluids in porous media. PhD-thesis University of Oklahoma (1964)

Gadiev, S.: Use of vibrations in oil production. Nedra Press (in Russian) (1977)

Neretin, V., Yudin, V.: Results of experimental study of the investigation of acoustic treatment on percolation processes in saturated porous media, in 'Topics in non-linear geophysics', All-Union Research Institute of Nuclear Geophysics and Geochemistry (VNIIYaGG), pp 132-137 (1981) 
Beresnev, I., Johnson, P.: Elastic wave stimulation of oil production: a review of methods and results. Geophysics 59, 1000-1017 (1994)

Poesio, P., Ooms, G., Schraven, A.P., Bas, F.v.d.: Theoretical and experimental investigation of acoustic streaming in porous material. Phys. Rev. E 66(1) ( July 2002) art. no: 016309 part 2

Poesio, P., Ooms, G., Barake, S., Bas, F.v.d.: An investigation of the influence of acoustic waves on the liquid flow through a porous material. J. Acous. Soc. Am. 111, 2019-2025 (2002) 